\title{
Identification en géotechnique par une méthode basée sur le théorème des travaux virtuels
}

\section{DENG}

Département des CGM

Mines

École polytechnique

de Montréal

CP 6079

Succ. Centre-ville

Montréal QC

Canada $\mathrm{H} 3 \mathrm{C} 3 \mathrm{~A} 7$

\section{NGUYEN-MINH}

Laboratoire de Mécanique des Solides

École polytechnique 91128 Palaiseau France

\begin{abstract}
Une nouvelle méthode de rétro-analyse basée sur le théorème des travaux virtuels a été proposée, avec l'établissement d'un nouveau critère d'identification, la minimisation d'une fonction erreur basée sur le théorème des travaux virtuels. La méthode est simple et facile à programmer, tout en étant efficace et robuste. Elle permet d'identifier les paramètres mécaniques du matériau et les paramètres du chargement, et s'applique aux problèmes non linéaires élastoplastiques.

L'application de la méthode au cas réel illustre sa faisabilité pratique en géotechnique.
\end{abstract}

Mots-clés : identification de paramètres, rétro-analyse, caractêrisation, problème inverse, théorème des travaux virtuels, géotechnique.

\section{Identification in geotechnical engineering by a method based on the virtual work principle}

A new back analysis method based on the virtual work principle is proposed, with establishment of a new identification criterion. the minimization of the error on the virtual work principle. This method is simple and easy to program, efficient and robust. It allows to identify mechanical and loading parameters, and can be applied to non linear elasto-plastic problems. Application of this methode to a history case illustrate its practical feasibility in geotechnical engineering.

Key words: parameter identification, back-analysis, characterization, inverse problem, virtual work principle, geotechnical engineering. 


\section{1}

\section{Introduction}

Des moyens de calcul numérique variês sont couramment appliqués dans la pratique de l'ingénieur pour le dimensionnement des structures en géotechnique tels que tunnels, cavités souterraines, excavations minières, pentes, fondations de barrage, etc. Néanmoins, prédire le comportement mécanique des structures avec une précision suffisante n'est pas un travail facile. La fiabilité de la prédiction dépend entièrement de la précision des données d'entrée utilisées dans l'analyse. Malheureusement, il est extrêmement difficile de déterminer quantitativement, de manière suffisamment précise, les structures géologiques, les propriétés géomécaniques des matériaux in situ, l'état des contraintes initiales, etc. On observe donc souvent une différence entre le comportement réel des structures et la prédiction, même si des investigations géologiques, hydrogéologiques et géotechniques poussées, et des analyses complexes sur ordinateur ont été menées.

Pour surmonter cette difficulté, on peut utiliser des observations in situ pendant la construction des structures, pour réévaluer les données d'entrée des paramètres géologiques et géomécaniques. Une technique appelée rétro-analyse est à la clé de cette démarche. Elle peut permettre de diminuer l'écart entre prédiction et réalité par un étalonnage des paramètres (Sakurai, 1993).

La méthode de rétro-analyse a été introduite et développée en géotechnique il y a plus d'une vingtaine d'années (Gioda et Jurina, 1981; Cividini et al., 1981; Sakurai et Takeuchi, 1983; Gioda et Sakurai, 1987 ; Akutagawa, 1991; Yang, 1996). Mais il reste encore beaucoup d'améliorations à attendre, surtout pour les problèmes non linéaires.

En général, la rétro-analyse applique le même modèle numérique que celui utilisé lors de l'analyse des contraintes dans un processus itératif d'optimisation. La procédure d'itération permet de corriger des valeurs d'essai des paramètres inconnus de sorte que l'écart

$$
F=\left\{\sum_{i=1}^{n}\left[u_{i}(\mathrm{P})-u_{i}\right]^{2}\right\}^{1 / 2}
$$

entre les mesures $u^{*}$ et les valeurs calculées $u$ soit minimisé ( $\mathrm{P}$ est le vecteur de paramètres à identifier, $n$ est le nombre des mesures). L'équation (1) est la fonction d'erreur (ou fonctionnelle cout) du problème de minimisation.

Comme l'expression analytique de l'écart $F$ ne peut pas être définie, l'algorithme de minimisation ne doit pas exiger l'évaluation analytique du gradient de la fonction (Cividini et al, 1981). Même si le problème est linéaire, l'écart $F$ défini par l'équation (1) est une fonction non linéaire complexe des quantités inconnues (Sakurai et al., 1994).

En général, des algorithmes utilisant des dérivés de la fonction d'erreur sont réputés être plus efficaces que ceux qui se sont basés seulement sur des valeurs de la fonction d'erreur (Press et al., 1992; Ledesma et al., 1996). Il est donc important de noter que l'efficacité de la rétro-analyse dépend largement du choix de la fonctionnelle coût. Récemment, on a cherché à établir de nouveaux critères d'identification dont les dérivées par rapport aux inconnues sont explicites. Par exemple, une méthode d'identification des modules élastiques des milieux continus fondée sur la minimisation sur l'erreur en loi de comportement a été proposée dans Constantinescu (1994).

Pour des problèmes non linéaires élastoplastiques, un nouveau critère d'identification basé sur le théorème des travaux virtueis, ainsi qu'une nouvelle méthode de rétro-analyse sont proposés dans ce travail.

\section{2}

\section{Principe de la méthode}

Pour présenter la nouvelle méthode de rétro-analyse, on doit définir deux modèles: un modèle de référence qui est la modélisation du problème réel (problème direct), et un modèle modifié qui est le problème traité avec des paramètres inconnus dans le système et qui inclut les mesures imposées sur la frontière (problème inverse). Par comparaison entre ces deux modèles, une méthode de rétro-analyse peut être obtenue.

\section{2}

\section{Modèle de référence}

Une structure occupant le domaine $\Omega$ de frontière $\Gamma$ (Fig. 1), est caractérisé à l'instant $t=0$ par son état initial. Pendant un intervalle de temps $[0, t]$, la structure subit une évolution suivant un certain trajet de chargement, qui se traduit par une histoire des conditions aux limites. Si la loi de comportement de la structure est bien identifiée, on peut alors calculer tous les champs mécaniques dans la strucutre pendant l'intervalle de temps $\left[0, t_{f}\right]$, et en particulier à l'état final $t_{f}$

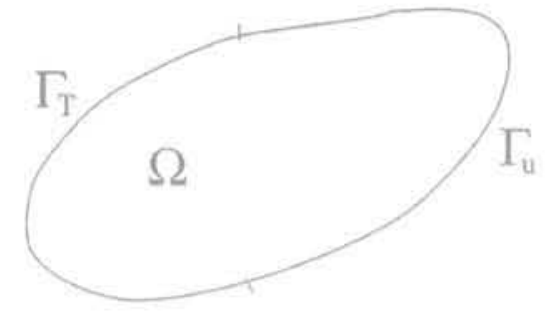

FIG.1 Modèle de référence.

Reference model.

Précisons maintenant les hypothèses effectuées ainsi que les notations. On se place dans le cadre de petites perturbations isothermes et quasistatiques. On définit $\mathbf{u}(\chi, t), \varepsilon(\chi, t)$ et $\sigma(\chi, t)$ les champs de déplacements, de déformations et de contraintes respectivement, qui dépendent du point $\chi \in \Omega$ et du temps $t \in[0, t]$.

L'état initial, considéré comme l'état de référence pour les déplacements, est l'état naturel défini par

$$
\left\{\begin{array}{l}
u(x, 0)=0 \\
\sigma(x, 0)=\sigma_{0} \quad x \in \Omega
\end{array}\right.
$$

Soit $\mathbf{n}$, la normale à $\Gamma$ extérieure à $\Omega$. La frontière $\Gamma$ est partitionnée en deux sous-ensembles $\Gamma_{\nu}$ et $\Gamma_{T}$ disjoints avec $\Gamma_{u}$ non nul, les déplacements étant imposés sur et $\Gamma_{\nu}$ et les efforts extérieurs $T(\chi t)$ étant imposés sur $\Gamma_{T}$. Les équations du problème d'évolution seront écrites sur les dérivées temporelles des champs mécniques, notées $\left({ }^{\circ}\right)$. 
Le champ des déplacements doit être cinématiquement admissible avec la condition aux limites sur la frontière $\Gamma_{u}$ :

$$
\left\{\begin{array}{l}
\varepsilon(x, t)=\frac{t}{2}\left(\nabla u(x, t)+\nabla^{\top} u(x, t)\right) \quad x \in \Omega \quad t \in\left[0, t_{f}\right] \\
u(x, t)=u^{d}(x, t) \quad x \in \Gamma_{u} \quad t \in\left[0, t_{f}\right]
\end{array}\right.
$$

Le champ des contraintes doit être statiquement admissible avec la condition aux limites sur la frontière $\Gamma_{\tau}$ :

$$
\left\{\begin{array}{lll}
\operatorname{div} \sigma(x, t)+p \mathbf{f}(x, t)=0 & x \in \Omega & t \in\left[0, t_{f}\right] \\
\dot{\sigma}(x, t), \mathrm{n}=\mathrm{T}^{d}(x, t) & x \in \Gamma_{T} & t \in\left[0, t_{f}\right]
\end{array}\right.
$$

$\rho$ étant la masse volumique, $\mathrm{f}$ étant la force massique.

Les données $\mathbf{u}^{d}$ et $\mathbf{T}^{d}$ satisfont initialement

$$
\begin{cases}u^{d}(x, 0)=0 & x \in \Gamma_{u} \\ T^{d}(x, 0)=\sigma_{0} & \text { n } \quad x \in \Gamma_{T}\end{cases}
$$

On note $\mathrm{T}_{0}=\mathrm{T}^{\mathrm{d}}(\alpha, 0)$ dans la suite du texte.

L'état initial (2), les conditions cinématiques (3) et les conditions statiques (4), définissent un problème d'évolution bien posé (modèle de référence), c'est-à-dire dont la solution pour le couple $(\sigma, \varepsilon)$ existe et est unique dans $\Omega$.

Pour simplifier l'écriture, on suppose

$$
\left\{\begin{array}{l}
\dot{u}^{d}(x, t)=0 x \in \Gamma_{u}, \quad t \in\left[0, t_{f}\right] \\
\dot{f}(x, t)=0 \quad x \in \Omega \quad t \in\left[0, t_{f}\right]
\end{array}\right.
$$

c'est un cas très général en géotechnique.

Notons $u_{r}, \varepsilon$, et $\sigma$ les solutions du modèle de référence (solutions réelles) à l'instant $t_{p}$, avec la force extérieure $\mathrm{T}_{t r}$ En prenant $\mathbf{u}_{r}$ et $\varepsilon_{r}$ respectivement comme champ de déplacement virtuel et champ de déformation virtuel, on applique le théorème des travaux virtuels (TTV) pour les champs de contrainte $\sigma_{i}$, on obtient la formule suivante:

$$
\int_{\Omega}\left(\sigma_{r}-\sigma_{0}\right): \varepsilon_{r} d x-\int_{\Gamma_{T}}\left(T_{t_{T}}-\mathbf{T}_{0}\right) \cdot \mathbf{u}_{r} d x=0
$$

Cette équation est toujours vérifiée pour les solutions réelles et est indépendante de la loi de comportement.

\section{2}

\section{Modèle modifié}

Dans le problème inverse que nous considérons, le domaine $\Omega$ et les sous-ensembles $\Gamma_{T}$ et $\Gamma_{\text {u }}$ de $\Gamma$ sont déterminés, de mème que l'intervalle de temps $\left[0, t_{t}\right]$. L'état initial est l'état naturel (2). En revanche, on ne connaît pas certains paramètres de la loi de comportement du matériau et/ou certains paramètres de chargement, et on suppose qu'on dispose de mesures du déplacement dans l'état final, notées $\mathbf{u}^{m}$, sur une partie $\Gamma_{T}^{u}$ de $\Gamma_{T}, \subset \Gamma_{T}^{\mu}$ (Fig. 2).

$$
\mathbf{u}\left(x, t_{f}\right)=\mathbf{u}^{m} x \in \Gamma_{T}^{u}
$$

Ainsi, le domaine $\Omega$ de la structure dans le modèle modifié reste identique à celui du modèle de référence, mais ses conditions aux limites ont été modifiées pour

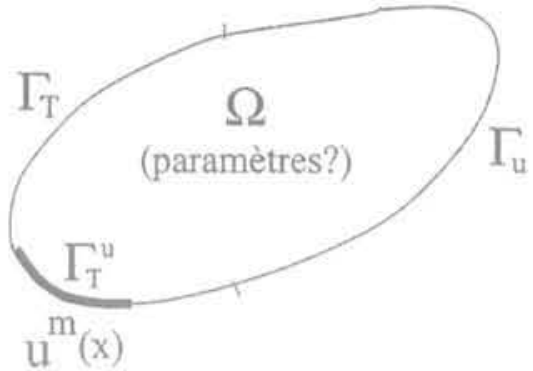

Fig. 2 Modèle modifié. Modified model.

tenir compte des observations. La frontière des déplacements donnés pour le modèle modifié à l'instant $t_{f}$ est changée en $\left(\Gamma_{u} \cup \Gamma_{T}^{u}\right)$, et celle relative aux forces est donc devenue $\left(\Gamma_{T} \backslash \Gamma_{T}^{u}\right)$. Le problème modifié est défini comme suit:

$$
\left\{\begin{array}{l}
\dot{\varepsilon}(x, t)=\frac{1}{2}\left(\nabla \mathbf{u}(x, t)+\nabla^{T} \mathbf{u}(x, t)\right) x \in \Omega t \in\left[0, t_{f}\right] \\
\operatorname{div} \sigma(x, t)=0 x \in \Omega t \in\left[0, t_{f}\right] \\
\sigma(x, t) \cdot \mathbf{n}=\dot{T}^{d}(x, t) \quad x \in\left(\Gamma_{T} \backslash \Gamma_{T}^{u}\right) \quad t \in\left[0, t_{f}\right] \\
\mathbf{u}\left(x, t_{f}\right)=\mathbf{u}^{m} x \in \Gamma_{T}^{u}
\end{array}\right.
$$

avec la loi de comportement définie dans le modèle de référence.

\section{3}

\section{Principe de la rétro-analyse}

L'objet de la rétro-analyse est l'identification des paramètres mécaniques du matériau, et des paramètres de chargement, de manière à ce que ces paramètres soient compatibles avec les efforts imposés sur $\Gamma_{T}$, ainsi qu'avec les déplacements finaux $\mathbf{u}^{m}$ observés sur $\Gamma_{T}^{4}$.

La résolution du modèle modifié, pour des valeurs arbitraires données des paramètres inconnus et des déplacements $\mathbf{u}^{m}$ imposés, donne à l'instant $t_{t}$ les solu-

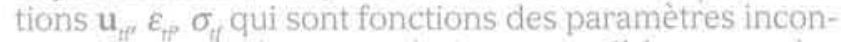
nus. Ces solutions seraient compatibles avec les mesures données $\mathbf{u}^{m}$ sur $\Gamma_{T}^{\mu}$ et les efforts imposés sur $\left(\Gamma_{T}, \Gamma_{T}^{\mu}\right)$, mais pas nécessairement avec les conditions aux limites du modèle de référence (avec les efforts imposés sur $\Gamma_{T}$ ).

Afin de tendre vers les solutions du modèle de référence, les paramètres inconnus dans le modèle modifié doivent être choisis de sorte que les champs de déplacements $u$, de déformations $\varepsilon$ et de contraintes $\sigma$ des solutions du modèle modifié satisfassent au mieux aux conditions aux limites du modèle de référence.

Pour réaliser la rétro-analyse des paramètres. $\mathrm{P}$, avec les conditions aux limites en force du modèle de référence, on définit sur le modèle modifié une erreur en TTV, écrit à l'instant $t_{f}$ :

$$
\mathrm{F}\left(\mathrm{P}, \mathrm{u}_{i f}, \varepsilon_{i f}, \sigma_{i f}\right)=\left[\int_{\Omega}\left(\sigma_{i f}-\sigma_{0}\right): \varepsilon_{i f} d x-\int_{\Gamma_{T}}\left(\mathrm{~T}_{t_{f}}-\mathrm{T}_{0}\right) \cdot \mathrm{u}_{t_{f}} d x\right]^{2}
$$

Le critère de la rétro-analyse est la minimisation de la fonctionnelle $\mathrm{F}$ qui représente l'erreur en TTV. On souligne que $u_{i p} \in_{\mathbb{p r}} \sigma t_{f}$ sont solutions du modèle modi- 
fié à l'instant $t_{f}$ (avec des mesures imposées) et sont fonctions implicites des paramètres $\mathrm{P}, \mathrm{T} t_{f}-\mathrm{T}_{0}$ sont les efforts extérieurs du modèle de référence, soit:

$$
\mathrm{T}_{\text {if }}-\mathrm{T}_{0}=\left(\sigma_{r}-\sigma_{0}\right) \cdot \mathrm{n} \text { sur } \Gamma_{T}
$$

Pour minimiser efficacement la fonctionnelle coût (10), on doit généralement chercher à en obtenir les dérivées par rapport aux paramètres inconnus. Mais il n'est pas facile d'obtenir ces dérivées analytiquement. D'après le principe de minimisation en utilisant les dérivées, on cherche les paramètres qui satisfont l'équation (12), cela rend l'erreur F minimale (on a omis l'indice $t_{f}$ pour simplifier l'écriture).

$$
\int_{\Omega}\left(\sigma-\sigma_{0}\right): \varepsilon d x-\int_{\Gamma T}\left(\mathrm{~T}-\mathrm{T}_{0}\right) \cdot u d x=0
$$

On retrouve l'équation de base (12) que nous allons utiliser pour la rétro-analyse,

Les deux termes de l'équation (12) ont des interprétations physiques évidentes:

$$
W=\int_{\Omega}\left(\sigma-\sigma_{0}\right): \varepsilon d x
$$

est le travail des efforts intérieurs du modèle modifié dans le domaine $\Omega$ et

$$
\Phi=\int_{r_{T}}\left(T-T_{0}\right) \cdot u d x
$$

est le travail des efforts extérieurs sur le champ $\varepsilon$ du modèle modifié.

Imaginons que l'on puisse exprimer l'équation de base (12) sous une forme explicite des paramètres $P$ :

$$
F(\mathbf{P}, \sigma, \varepsilon, \mathrm{u})=0
$$

Puisque les champs $\sigma$, $\varepsilon$ et $u$ sont fonctions des paramètres, cette expression fournit en effet une relation entre les valeurs des paramètres réactualisés $\mathrm{P}^{n-1}$ et les précédentes $\mathrm{P}^{\mathrm{i}}$, comme suit:

$$
F\left(\mathrm{P}^{n+1}, \sigma\left(\mathrm{P}^{n}\right), \varepsilon\left(\mathrm{P}^{n}\right), u\left(\mathrm{P}^{n}\right)\right)=0
$$

$n$ étant le nombre de pas du calcul.

En général, la résolution de l'équation (16) ressemble à un problème de type point fixe (Arnaudiès et Fraysse, 1989), qui nécessite une procédure d'itération qu'on supposera converger. Comme la méthode est basée sur le TTV, elle revêt un caractère intrinsèque qui lui confère robustesse et permet son application à des problèmes non linéaires élastoplastiques. Comme une série de mesures donne seulement une relation entre les nouvelles valeurs des paramètres $\mathrm{P}$ et les précédentes, on a besoin de $N$ séries de mesures pour rétroanalyser $N$ paramètres simultanément.

L'algorithme de la rétro-analyse pour un seul paramètre est établi comme suit:

1) donner la valeur initiale du paramètre $p^{0}$;

2) résoudre le problème modifié, avec les mesures données $\mathbf{u}^{m}$ sur $\Gamma_{T}^{u}$, par exemple en utilisant un code d'éléments finis, obtenir les solution $\sigma^{n}, \varepsilon^{n}$ et $\mathbf{u}^{n}$, ( $n$ est le nombre d'itération);
3) calculer la nouvelle valeur du paramètre $p^{n+1}$ en résolvant l'équation (16) avec une méthode numérique ou dans quelques cas avec une solution explicite;

4) contrôler la convergence d'itération d'après un critère d'arrêt, par exemple par une tolêrance de la différence entre la nouvelle valeur du paramètre et la précédente, si la tolérance n'est pas atteinte, retourner au deuxième pas avec la nouvelle valeur du paramètre. Pour $N$ paramètres à identifier, avec $N$ mesures, on dispose de $N$ problèmes modifiés. On peut ainsí établir l'algorithme général de la même manière, c'est un algorithme à directions alternées (Constantinescu, 1994).

\section{3}

\section{Identification et validation}

Dans ce qui suit, on donne quelques exemples d'expressions explicites des paramètres inconnus à partir de l'équation (15) utilisées pour rétro-analyser des paramètres mécaniques ou de chargement, et l'on valide la méthode sur des exemple numériques. Les “mesures expérimentales » sont les données issues d'un calcul direct numérique préliminaire.

\section{1}

\section{Paramètres mécaniques}

\subsection{5 \\ Paramètres élastiques du matériau}

On considère un corps élastique qui occupe dans la configuration de référence le domaine régulier $\Omega$, avec une loi de comportement du matériau élastique homogène linéaire.

Dans le cas isotrope, compte tenu de l'état initial, la loi de comportement donne

$$
\sigma-\sigma_{0}=\frac{v E}{(1+v)(1-2 v)} \operatorname{tr} \varepsilon \mathbf{I}+\frac{E}{1+v} \varepsilon
$$

E étant le module de Young du matériau, $v$ le coefficient de Poisson, I le tenseur unitaire.

Compte tenu de cette équation, l'équation de base de rétro-analyse (12) devient

$$
\frac{v E}{(1+v)(1-2 v)} \int_{\Omega}(\operatorname{tr} \varepsilon)^{2} d x+\frac{E}{1+v} \int_{\Omega} \varepsilon: \varepsilon d x-\Phi=0
$$

Les valeurs réactualisées des paramètres élastiques peuvent être obtenues par la résolution de l'équation (18). Ainsi, pour la rétro analyse du module de Young, on obtient une solution explicite:

$$
E=\frac{\Phi}{\frac{v}{(1+v)(1-2 v)} \int_{\Omega}(\operatorname{tr} \varepsilon)^{2} d x+\frac{1}{1+v} \int_{\Omega} \varepsilon: \varepsilon d x}
$$

Pour la rétro-analyse de $v$, on peut résoudre l'équation (18) du second degré en $v$.

On peut de la même façon obtenir des expressions analytiques dans les cas de matériaux anisotropes. 
Validation : identification du module de Young

Considérons une structure hypothétique élastique linéaire homogène isotrope occupant un domaine $\Omega$ (Fig. 3)

On fait d'abord un calcul direct, avec des paramètres élastiques du matériau donnés comme $E=1 \times 10^{5}, v=0,1$. Si les déplacements sur le bord $B C$ du calcul direct sont utilisés comme données de mesure, la rétro-analyse du module de Young $E$ est faite par itération, La figure 4 montre la convergence d'itération du module de Young $E$ pour différentes valeurs initiales.

Après 3, 4 itérations le module calculé est à $5 \%$ du module réel. La figure 5 a illustre l'évolution de l'erreur $F$ en théorème des travaux virtuels du module à identifier. Dans la procédure de rétro-analyse, la diminution de l'erreur en théorème des travaux virtuels est très rapide (voir figure 5 b)

\section{3 sitis?}

\section{Paramètres plastiques du matériau}

Considérons maintenant un matériau élastoplastique parfait, dont on recherche à identifier les paramètres plastiques $\mathbf{P}$ selon un critères de plasticité $f$ :

$$
f(\sigma, \mathrm{P})=0
$$

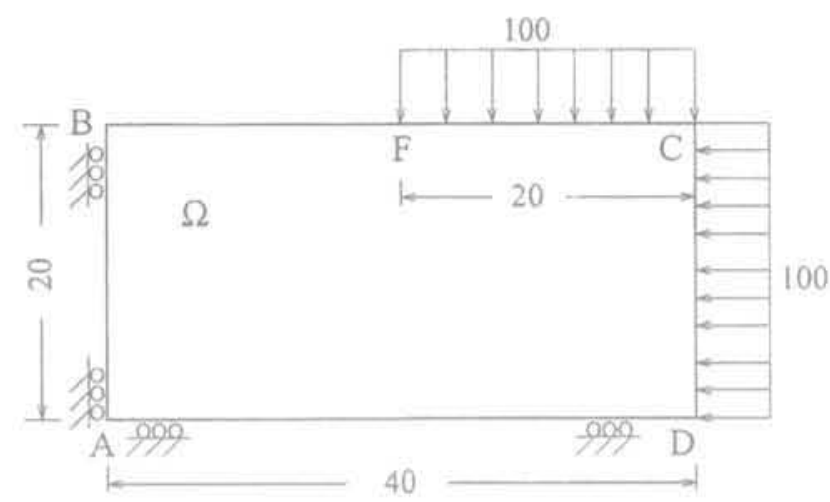

FIG. 3 Exemple numérique élastique. Numerical elastic example.

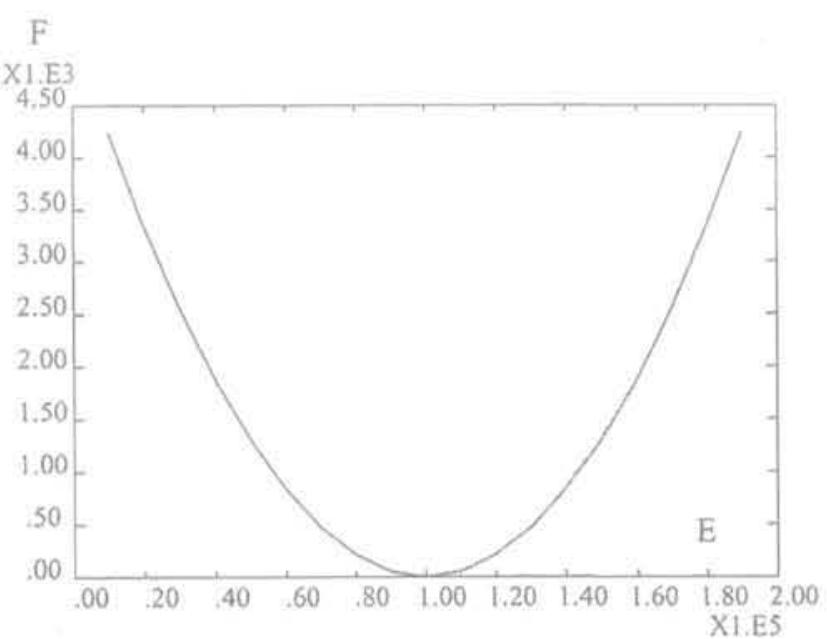

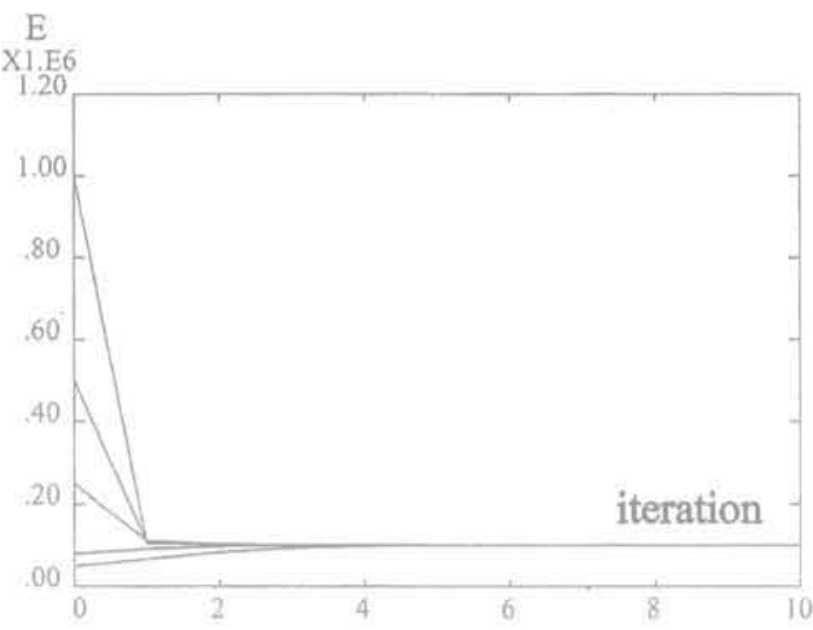

FG. 4 Convergence du module d'Young E pour différentes valeurs initiales: exemple numérique élastique.

Convergence of the Young's modulus E for different initial values: numerical elastic example.

Tenant compte du temps pour lequel apparait la plasticité, on peut obtenir une relation explicite entre la solution $\sigma$ et les paramètres de plasticité en utilisant le fait que le critère de plasticité est atteint.

Plus précisément, on procède comme suit: dans la résolution du problème modifié, on distingue la zone plastifiée $\Omega^{p}$ de la zone élastique $\Omega^{c}$ du domaine $\Omega$. Le travail des efforts intérieurs $W$ peut être calculé comme la somme de ses valeurs sur les deux zones $W=W^{c}+W^{p}$ (en notant la zone élastique par l'exposant e et la zone plastique par p). Dans la zone élastique $\Omega^{c}$, on peut calculer directement sa valeur $W^{\pi}$. Et dans la zone plastique $\Omega$, puisque $W$ est indépendant du repère, on peut exprimer son intégrale dans le repère des contraintes principales et ainsi obtenir une expression facilement utilisable pour la résolution de ce problème.

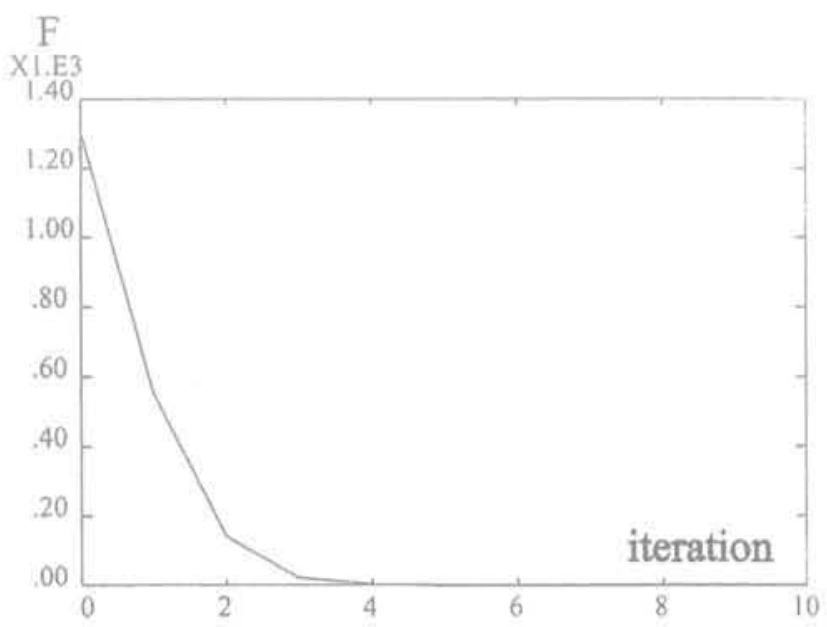

FIG. 5 Évolution de la fonction erreur: exemple numérique élastique, a) Évolution de l'erreur en TTV en fonction de la valeur du module; b) Diminution de l'erreur en TTV, valeur initiale du module d'Young $=0,5 \times 10^{5}$.

Evolution of the error function: numerical elastic example. a) Evolution of the error on TTV with the modulus value; $b$ ) Diminution of the error on TTV, initial value of the Young's modulus $=0.5 \times 10^{5}$ 
A titre d'illustration nous donnons ici des exemples pour les critères de plasticité de Tresca et de MohrCoulomb, on pourra procéder de même pour les autres critères courants utilisés par les ingénieurs. On note $\sigma_{i,}$ $\sigma_{2}$ et $\sigma_{3}$ les composantes des contraintes principales avec $\sigma_{1} \geq \sigma_{2} \geq \sigma_{i}, \varepsilon^{1}, \varepsilon^{2}$ et $\varepsilon^{3}$ composantes des déformations dans le repère des contraintes principales correspondant. On suppose $\sigma_{0}=0$ pour simplifier l'écriture (si $\sigma_{0} \neq 0$, il suffit de soustraire le terme $\int_{0} \sigma_{0}: \varepsilon d x$ de $W$ ),

- Critère de Tresca

Le critère de Tresca s'écrit dans notre cas:

$$
\sigma_{1}-\sigma_{3}=2 c
$$

$c$ étant la résistance en cission simple du matériau, soit

$$
\sigma_{1}-2 c=\sigma_{3}
$$

Dans la zone plastique $\Omega^{p}$, le critère est atteint, et on a:

$$
\begin{aligned}
W^{p}=\int_{\Omega^{p}} \sigma: \varepsilon d x & =\int_{\Omega^{p}}\left(\sigma_{1} \varepsilon^{1}+\sigma_{2} \varepsilon^{2}+\sigma_{3} \varepsilon^{3}\right) d x \\
& =2 c \int_{\Omega^{p}} \varepsilon^{1} d x+\int_{\Omega^{p}}\left(\sigma_{3} \varepsilon^{1}+\sigma_{2} \varepsilon^{2}+\sigma_{3} \varepsilon^{3}\right) d x
\end{aligned}
$$

Selon l'équation de base de rétro-analyse (12), on a

$$
2 C \int_{\Omega} \varepsilon^{3} d x+\int_{\Omega}\left(\sigma_{3} \varepsilon^{3}+\sigma_{2} \varepsilon^{2}+\sigma_{3} \varepsilon^{3}\right) d x+W^{e}-\Phi=0
$$

d’où

$$
c=\frac{\Phi-W^{e}-\int_{\Omega^{p}}\left(\sigma_{3} \varepsilon^{1}+\sigma_{2} \varepsilon^{2}+\sigma_{3} \varepsilon^{3}\right) d x}{2 \int_{\Omega^{p}} \varepsilon^{1} d x}
$$

- Critère de Mohr-Coulom

Pour le critère de Mohr-Coulomb

$$
\frac{1}{2}\left(\sigma_{1}-\sigma_{3}\right)=c \cos \phi-\frac{1}{2}\left(\sigma_{1}-\sigma_{3}\right) \sin \phi
$$

c étant la cohésion et $\phi$ étant l'angle de frottement interne du matériau. De même façon, on obtient

$$
\begin{aligned}
& \int_{\Omega^{p}} \frac{2 c \cos \phi+\sigma_{3}(1-\sin \phi)}{1+\sin \phi} \varepsilon^{1} d x+ \\
& \int_{\Omega^{p}}\left(\sigma_{2} \varepsilon^{2}+\sigma_{3} \varepsilon^{3}\right) d x+W^{c}-\Phi=0
\end{aligned}
$$

Pour rétro-analyser le paramètre $\phi$, il est nécessaire de résoudre l'équation ci-dessus avec une méthode numérique. Pour rétro-analyser le paramètre $c$, on a une solution explicite

$$
c=\frac{\Phi W^{e}-\int_{\Omega^{p}}\left[\sigma_{2} \varepsilon^{2}+\sigma_{3} \varepsilon^{3}+\frac{\sigma_{3}(1-\sin \phi)}{1+\sin \phi} \varepsilon^{1}\right] d x}{\frac{2 \cos \phi}{1+\sin \phi} \int_{\Omega^{p}} \varepsilon^{4} d x}
$$

Validation : identification du paramètre plastique du matériau avec le critère de plasticité de von Mises

Un tunnel (Fig. 6) profond est creusé dans un milieu continu élastoplastique isotrope homogène. On admet ici que les contraintes initiales sont isotropes et homogènes. On considère un problème en déformation plane. Le rayon du tunnel est $R=5 \mathrm{~m}$, et on considère un quart de la géométrie de $55 \mathrm{~m} \times 55 \mathrm{~m}$ pour tenir compte de la symétrie. Les paramètres élastiques du matériau sont $E=100 \mathrm{MPa}, v=0,2$. Comme critère de plasticité, on prend à titre d'illustration celuí de von Mises pour le massif, avec $c=10 \mathrm{KPa}$ dans le calcul direct pour obtenir des «mesures » de dépacement. Le chargement est appliqué sur la paroi du tunnel supposé sans soutènement, avec la valeur équivalant aux contraintes initiales $20 \mathrm{KPa}$, le rayon plastique du tunnel vaut $R_{p}=3,5 \mathrm{R}$ (la convergence plastique du tunnel $U$ est quatre fois plus grande que celle du tunnel supposé élastique $U_{\mathrm{e}}$ ).

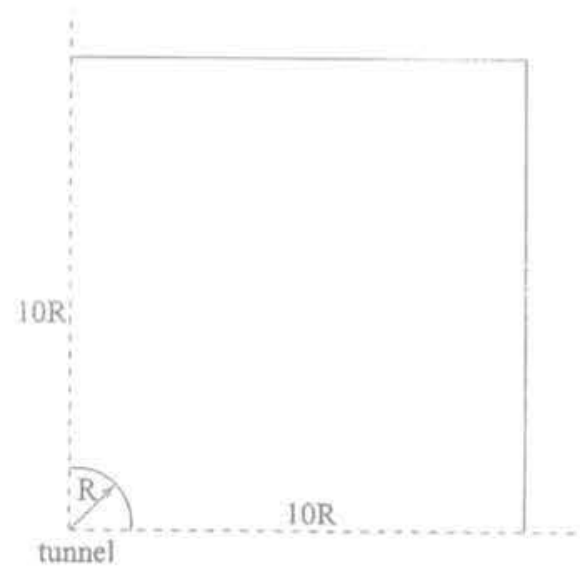

FG.6 Exemple numérique d'un tunnel profond en élasto-plasticité.

Numerical example of a deep tunnel in elastoplasticity.

En utilisant des déplacements sur une demi-partie de la paroi du tunnel comme données de mesure, le paramètre $c$ est identifiè $(=10,25 \mathrm{KPa})$ avec une très bonne convergence (Fig. 7). A cause d'une zone de plasticité étendue, le résultat donne une erreur de $2,5 \%$.

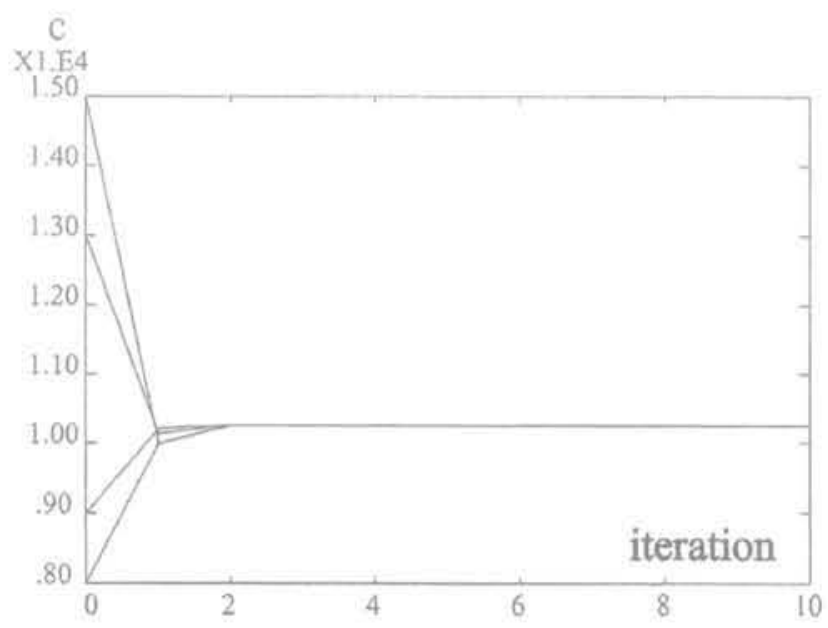

FIG. 7 Convergence de $c$ pour différentes valeurs initiales: exemple numérique d'un tunnel profond.

Convergence of $c$ for different initial values: numerical example of a deep tunnel. 
Pour vérifier la stabilité de la rétro-analyse, on affecte les "mesures» de bruits. Une erreur relative de $10 \%$ sur toutes les mesures de convergence se traduit par une erreur de $3,5 \%$ sur le paramètre identifié. On constate que la méthode de la rétro-analyse basée sur le TIV est très stable pour l'identification d'un seul paramètre, même si le matériau subit des déformations plastiques importantes.

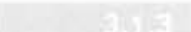

\section{Paramètres élastoplastiques de joints}

Le comportement d'un massif rocheux est parfois dominé par celui des joints et autres discontinuités (Deng et Nguyen-Minh, 2001). Dans le cas de joints élastoplastiques, des lois de comportement simples suffisent pour la pratique d'ingénieur. Il s'agit d'en identifier les paramètres.

Un joint $\Gamma$ sépare le domaine $\Omega$ en deux sous domaines $\Omega_{1}$ et $\Omega_{2}$ (Fig. 8), avec $\Omega=\Omega_{1} \cup \Omega_{2} \cup \Gamma_{\text {r }}$

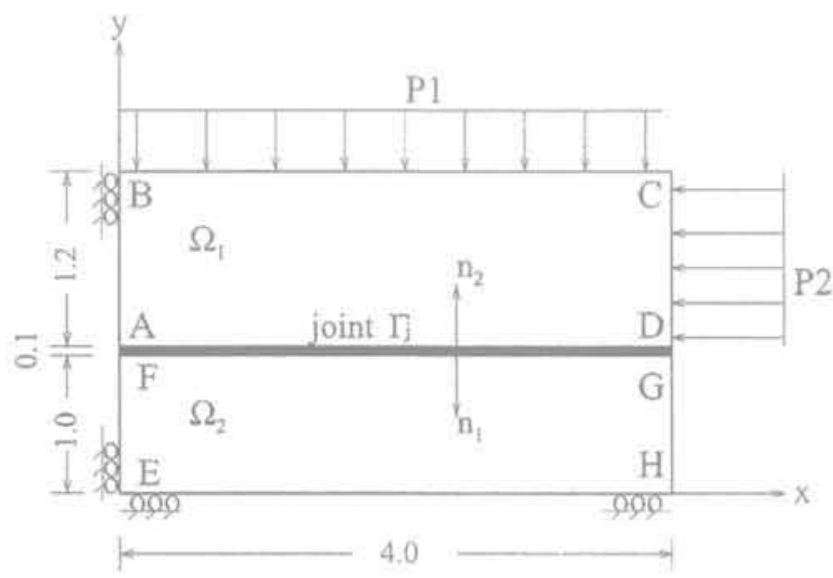

FIG. 8 Exemple numérique du problème ave joint.

Numerical example of joint problem

On considère le champ de déplacement continu et continüment différentiable sur deux volume $\Omega$, et $\Omega$, et discontinu au franchissement et de la surface $\Gamma$ frontière entre $\Omega_{1}$ et $\Omega_{2}$. On définit le saut du déplacement au franchissement du joint comme

$$
[[\mathbf{u}]]=\left(\mathbf{u}_{2}-\mathbf{u}_{1}\right) \mid \Gamma_{j}
$$

avec $u_{1}$ et $u_{2}$ champs de déplacement de $\Omega$, et $\Omega$, respectivement (en notant que le vecteur de la direction normale extérieure du joint $\mathrm{n}$ est celui de $\Omega$,. On écrit ausssi $[[\mathbf{u}]]$ selon les composantes normale $u_{n}$ et tangentielle $u_{s}$ du joint:

$$
[[\mathrm{u}]]=\left(u_{n}, u_{s}\right)^{T}
$$

et on note $\sigma_{\text {f }}$ et $\sigma$ les contraintes normale et tangentielle à l'interface des joints.

On considère une loi de comportement de Goodman, 1976) pour la réponse élastique et un critère de Mohr-Coulomb standard (Gudehus, 1977) pour simuler la réponse plastique. Le critère s'écrit:

$$
\sigma_{r}=c+\sigma_{n} \operatorname{tg} \phi
$$

$\sigma_{f}$ est la résistance de cisaillement du joint, $c$ la cohésion et $\phi$ l'angle de frottement interne du joint.

Pour la résolution du problème modifié, on distingue dans le joint $\Gamma$ la zone plastique $\Gamma^{p}$ de la zone élastique $\Gamma_{\text {i }}^{e}$. L'équation de base nous donne:

$$
\begin{aligned}
\int_{\Omega}\left(\sigma-\sigma_{0}\right): \varepsilon d x-\phi+\int_{\Gamma_{j}^{p}}\left(\sigma_{n}-\sigma_{n}^{0}\right) u_{n} d x \\
+\int_{\Gamma^{2}} k_{0} u_{n}^{2} d x+\int_{\Gamma_{j}^{2}} k_{s} u_{s}^{2} d x+\int_{\Gamma_{j}^{p}}\left(\sigma_{n} \operatorname{tg} \phi+c-\sigma_{s}^{0}\right) u_{y}^{2} d x=0
\end{aligned}
$$

avec $k$ et $k$ rigidités normale et tangentielle de joints,

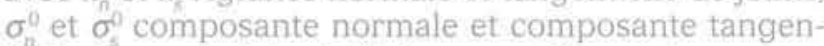
tielle des contraintes initiales respectivement.

Pour la rétro-analyse d'un seul paramètre, on isole ce paramètre, en supposant connus les autres paramètres. Ainsi, si on veut identifier $k_{c}$ d'après l'équation (31), on a:

$$
k_{\mathrm{s}}=\frac{\omega_{s}}{\int_{\Gamma_{\mathrm{f}}} u_{\mathrm{s}}^{2} d x}
$$

avec:

$$
\begin{gathered}
\omega_{s}=\Phi-\int_{\Omega}\left(\sigma-\sigma_{0}\right): \varepsilon d x-\int_{\Gamma j}\left(\sigma_{n}-\sigma_{n}^{0}\right) u_{n} d x- \\
\int_{\Gamma \rho_{j}^{p}}\left(\sigma_{n} \operatorname{tg} \phi+c-\sigma_{s}^{0}\right) u_{s} d x
\end{gathered}
$$

On procède de même pour identifier $k_{n}$, ou $\phi$, ou $c$.

Validation: identification des paramètres d'un joint en élasto-plasticité

On considère la structure hypothétique suivante : un domaine $\Omega$ partagé par un joint $\Gamma$ en deux sousdomaines $\Omega_{1}$ et $\Omega_{2}$, est chargé par une pression uniforme $P_{1}$ sur le bord $B C$ et une pression uniforme $P_{2}$ sur $C D$ (Fig. 8).

On considère les domaines $\Omega$, et $\Omega$, élastiques linéaires et le joint $\Gamma$ élastoplastique de type Mohr-Coulomb standard. On fait d'abord un calcul direct pour obtenir les déplacements qui seront utilisés ensuite comme données de mesure pour la rétro-analyse. Les paramètres du calcul direct sont: $E=1000,0, v=0,1$ sur $\Omega_{1}$ et $\Omega_{2} ; k_{n}=1,0 \times 10^{5}, k_{s}=10,0, c=2,0, \phi=45^{\circ}$ sur $\Gamma_{j}$, avec les chargements $P_{1}=9,0, P_{2}=400,0$ à l'instant $t_{0}$, et $P_{1}=9,0, P_{2}=650,0$ à l'instant $t_{f}$

On considère ici la rétro-analyse d'un seul paramètre et aussi de deux paramètres simultanément.

Soit à rétro-analyser un seul paramètre, la rigidité tangentielle du joint. Ón utilise les déplacements du calcul direct à l'instant $t_{0}$ ( $30 \%$ des éléments joints plastifiés) sur le bord $B C$ pour la rétro-analyse. La figure 9 donne la convergence d'itération de rétro-analyse de $k_{c}$ pour différentes valeurs initiales. On peut voir que la rétro-analyse converge très rapidement, 3-4 itérations sont suffisantes pour une résolution à $5 \%$ de la valeur réelle.

Soit à rétro-analyser deux paramètres simultanément, la cohésion $c$ et l'angle de frottement interne $\phi$ du joint, dans une structure plastifiée. Dans ce cas, deux séries de mesures, les déplacements du calcul sur le bord $B C$ à l'instant $t_{0}$ et à l'instant $t_{f}$ (avec $50 \%$ des éléments joints plastifiés) sont sélectionnés comme données de mesures pour identifier les deux paramètres $c$ et $\phi$. La figure 10 montre les processus d'itéra- 


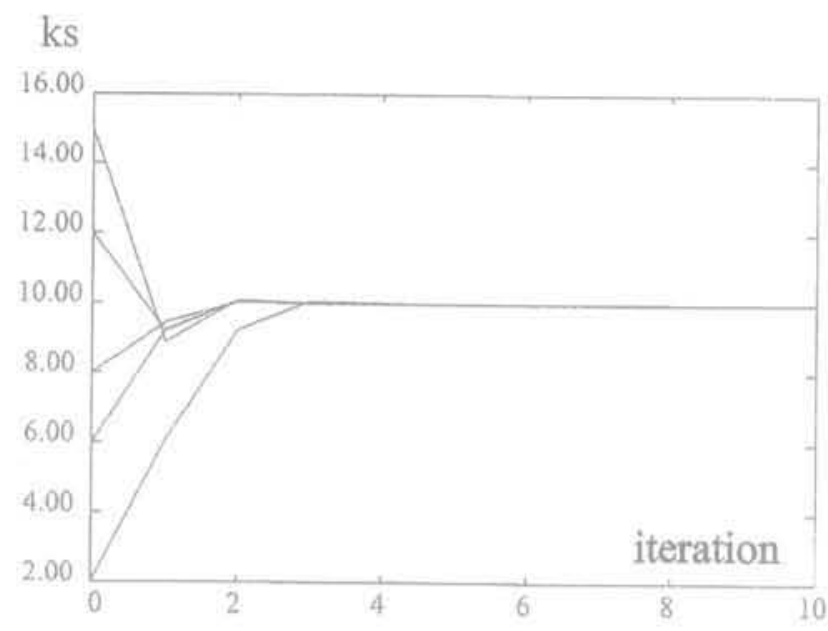

คG. 9 Convergence de $k$, pour différentes valeurs initiales: exemple numérique de joint.

Convergence of $k$ for different initial values numerical example of joint.
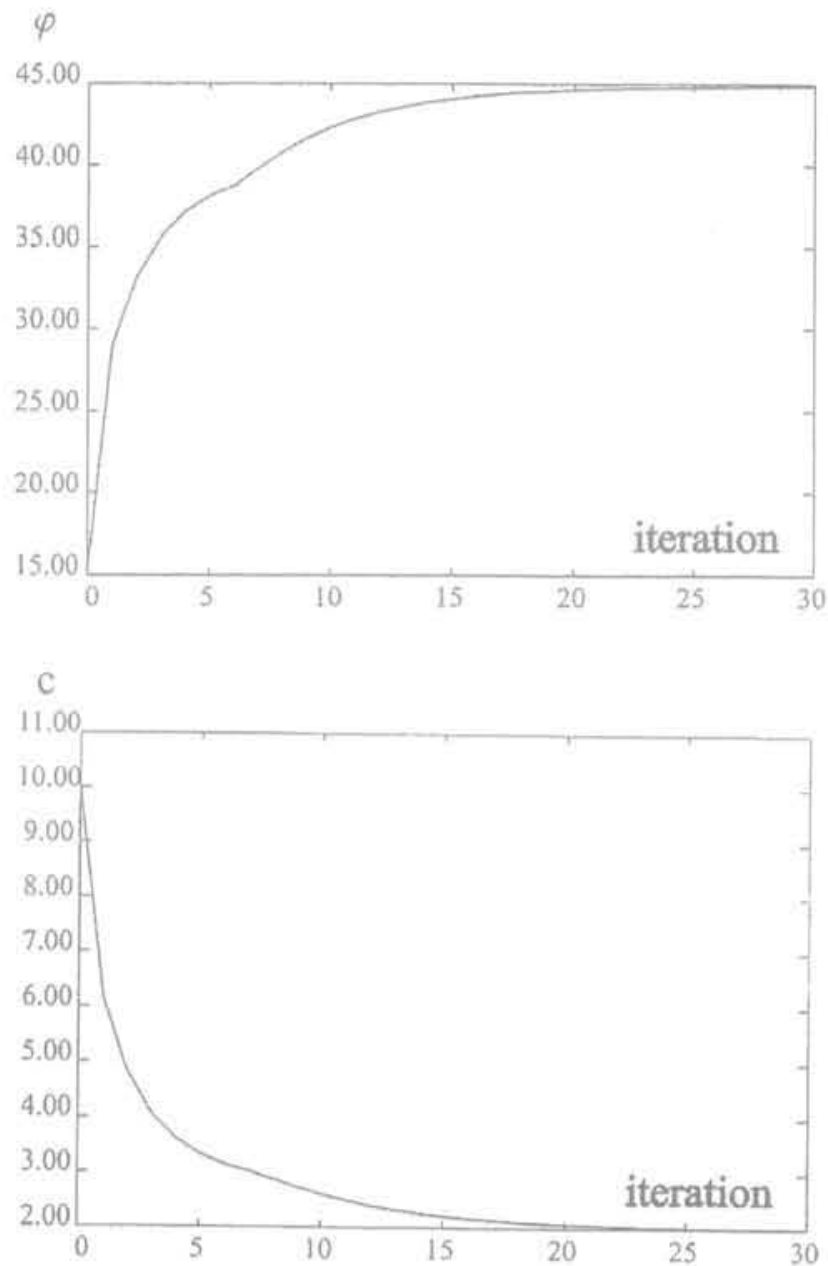

FIG. 10 Convergence de $\phi$ et $c$ du joint : exemple numérique de joint.

Convergence of $\phi$ and $c$ of the joint : numerical example of joint. tion de la rétro-analyse avec des valeurs initiales: $\phi=15^{\circ}, c=10$. Après 15, 20 itérations, les paramètres sont identifiés avec moins de $5 \%$ d'erreur.

3.2

\section{Paramètres de chargement pour un tunnel peu profond}

Les paramètres de chargement sont faciles à exprimer dans le terme du travail des efforts extérieurs $\Phi$. Avec l'équation de base de la rétro-analyse (12), ces paramètres peuvent ètre rétro-analysés facilement. indépendamment de la loi de comportement. Pour illustration, on montre la rétro-analyse des paramètres de chargement dans le creusement d'un tunnel de section circulaire, peu profond, modélisé en déformation plane par la méthode convergence confinement.

Soit un tunnel de contour $\Gamma_{\text {, }}$ creusé dans un massif semi-infini $\Omega$. Selon la méthode convergence confinement (AFTES, 1979; Panet, 1995), le creusement du tunnel peu profond peut être simulé par l'application d'une contrainte fictive $\sigma_{f}$ en paroi du tunnel et diminuant au fur et à mesure du creusement (Shahrour and Ghorbanbeigi, 1996; Deng et al., 1999).

$$
\sigma_{f}=(1-\lambda) \sigma_{0}
$$

$\lambda$ est appelé le taux de déconfinement variant de zéro á 1 au cours du creusement du tunnel, et égale $\lambda_{0}$ au moment $t_{0}$ oú le soutènement est mis en place.

Les efforts fictifs sur le contour du tunnel $\Gamma_{t u}$ sont donnés pour différents instants comme suit:

$$
T(x, t)= \begin{cases}\sigma_{0} \cdot n & t=0 \\ \left(1-\lambda_{0}\right) \sigma_{0} \cdot n & t=t_{0} \\ 0 & t=t_{t}\end{cases}
$$

En considérant l'intervalle de temps [0, $t]$, et en sup. posant que les mesures de déplacement a l'instant $t$. sont données comme $\mathbf{u}^{m}$ sur $\Gamma^{\nu}$, la résolution du problème modifié donne à l'instant $t$ les champs de déplacement $u$, de déformation $\varepsilon$ et de contraintes $\sigma$.

Pour un tunnel peu profond, on admet que les contraintes initiales sont non homogènes et anisotropes. Si on suppose par exemple comme dans les cas courants que les contraintes initiales $\sigma_{0}$ à une profondeur $h$ sont caractérisées par une composante principale verticale $\sigma$ connue, et une composante principale horizontale $\sigma_{h}$, telle que

$$
\sigma_{i}=\gamma h \quad \sigma_{h}=k_{0} \sigma_{4}
$$

avec $k_{0}$ coefficient de pression des terres au repos, en général indéterminé. Les paramètres $\lambda_{0}$ et $k_{0}$ sont paramètres de chargement dans ce problème.

Ainsi à l'instant $t_{p}$ le travail des efforts extérieurs du modèle de référence sur le champ $\varepsilon$ du problème modifié est

$$
\begin{aligned}
\Phi=\int_{\Gamma_{\mathrm{t} u}}- & \lambda_{0}\left(\sigma_{0} \cdot \pi\right) \cdot u d x \\
& =\int_{\Gamma_{t u}}\left(k_{0} \lambda_{0} \sigma_{v} \cos \theta u_{h}+\lambda_{0} \sigma_{v} \sin \theta u_{v}\right) d x
\end{aligned}
$$

avec $u_{h} u_{y}$ composantes horizontales et verticales de déplacement en paroi du tunnel, $\theta$ l'angle du rayon par rapport à l'axe horizontal.

En portant cette équation dans l'équation de base de la rétro-analyse (12), on a: 


$$
k_{0}=\frac{W-\lambda_{0} \int_{\Gamma_{u i}} \sigma_{v} \sin \theta u_{v} d x}{\lambda_{0} \int_{\Gamma_{t u}} \sigma_{v} \cos \theta u_{h} d x}
$$

ou

$$
\lambda_{0}=\frac{W}{\int_{r_{t u}}\left(k_{0} \sigma_{v} \cos \theta u_{h}+\sigma_{v} \sin \theta u_{i}\right) d x}
$$

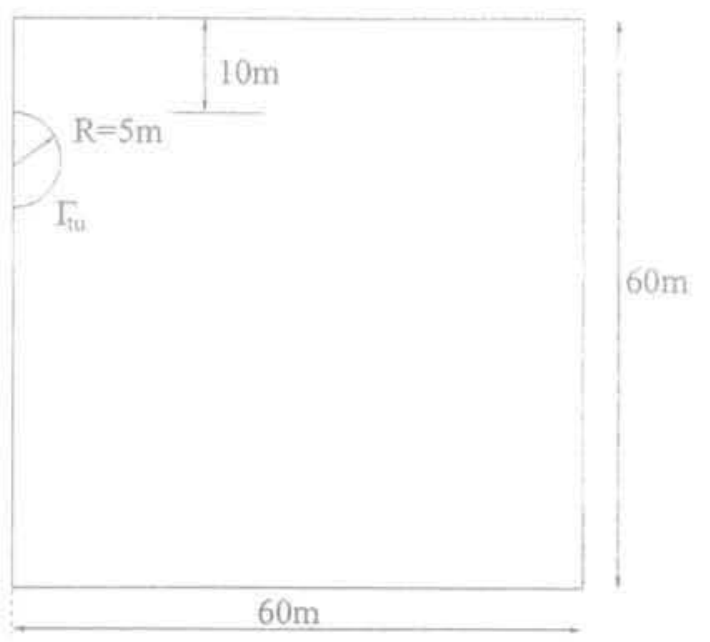

FIG,11 Exemple numérique d'un tunnel peu profond.

Numerical example of a shallow tunnel.

Validation : rétro-analyse de $\lambda_{0}$ et $k_{0}$

Un tunnel à faible profondeur est creusé dans un massif isotrope homogène élasto-plastique (Fig. 11). Compte tenu de la symétrie, on étudie une demi-partie de la géométrie.

tABLEAU1 Paramètres des matériaux: exemple numérique d'un tunnel peu profond. ( $\gamma$ : poids volumique)

\begin{tabular}{l|c|c|c|c|c} 
Matériaux & E (MPa) & $v$ & $c(\mathrm{KPa})$ & $\phi\left({ }^{\circ}\right)$ & $\gamma\left(\times 10^{4} \mathrm{~N} / \mathrm{m}^{3}\right)$ \\
Massif & 100,0 & 0,35 & 200,0 & 5.0 & 2,0 \\
\hline Béton & 15000,0 & 0,25 & & & 2,4 \\
\hline
\end{tabular}

On choisit pour le massif un modèle élasto-plastique parfait standard de Drucker-Prager. Le soutenement (épaisseur: $0,3 \mathrm{~m}$ ) est en béton, avec une loi de comportement élastique linéaire. Les paramètres des matériaux sont listés dans le tableau 1.

On va d'abord faire un calcul direct en se donnant tous les paramètres, $k_{0}=0,8, \lambda_{0}=0,45$. Ensuite, les déplacements du calcul direct servent de données de mesure pour rétro-analyser le taux de déconfinement $\lambda_{v}$ et le coefficient $k_{0}$ supposés inconnus.

Après le calcul direct, on obtient la convergence de la paroi du tunnel à l'instant $t_{0}$ et $t_{\text {. }}$. Pour identifier un seul paramètre, on utilise la convergence sur une demipartie (partie haute) de la paroi à l'instant $t_{0}$ comme données de mesures $\mathbf{u}^{n \text { in }}$, pour rétro-analyser le taux de déconfinement $\lambda_{v}$ et le coefficient $k_{0}$.

Les figures $12 \mathrm{a}$ et $12 \mathrm{~b}$ montrent la convergence de $\lambda_{0}$ et de $k_{0}$ pour différentes valeurs initiales. On peut constater que la convergence des itèrations est très rapide. Des valeurs initiales de $\lambda_{i}$ sont choisies dans l'intervalle 0,25 à 0,65 , et celles de $k_{0}$ dans 0,1 à 1,4 . Après 3, 4 itérations, $\lambda_{0}$ (ou $k_{0}$ ) est obtenu à $5 \%$ près.

On utilise ensuite $\mathbf{u}^{m}$ et la différence de déplacement entre les instants $t_{f}$ et $t_{0}$ sur la demi-partie de la paroi $\Delta \mathbf{u}^{m}$, pour rétro-analyser $\lambda_{n}$ et $k_{0}$ simultanément. La figure 13 donne les convergences de $\lambda_{0}$ et $k_{0 \gamma}$ avec des valeurs initiales: $\lambda_{0}=0,4, k_{0}=1,5$. Il faut 10 itérations pour obtenir les valeurs à $5 \%$ près.

La figure 14 montre le chemin de l'algorithme de la rétro-analyse à la surface de la fonctionnelle coùt $F$ repérée par les contours des isovaleurs. La figure a et la figure b sont respectivement relatives au premier problème modifié (â l'instant $t_{0^{\prime}}$ avec la mesure $\mathrm{u}^{\mathrm{m}}$ ) et au deuxième (à l'instant $t$, avec la mesure $\Delta \mathbf{u}^{m}$ ). On voit que l'erreur en TTV diminue à chaque itération, ce quí assure l'efficacité de la rétro-analyse.

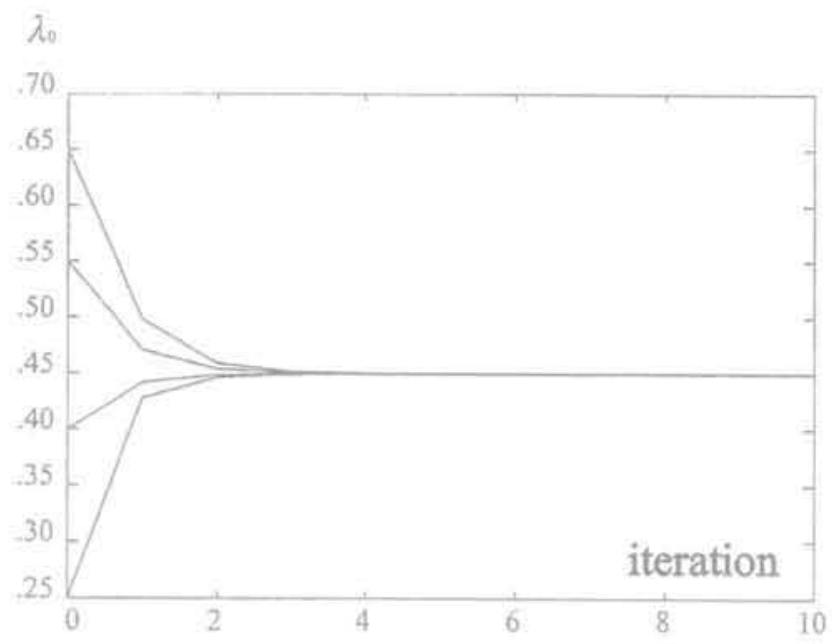

$k_{0}$

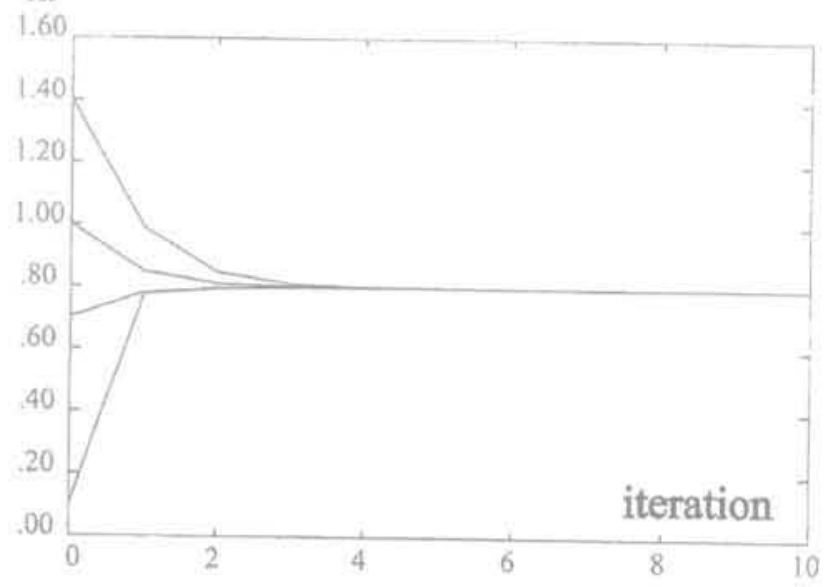

FIG. 12 Rétro-analyse d'un seul paramètre $\left(\lambda_{0}\right.$ ou $\left.k_{0}\right)$ : exemple numérique d'un tunnel peu profond. a) Rétro-analyse de $\lambda_{0}$; b) Rétroanalyse de $k_{0}$.

Back analysis of one parameter $\left(\lambda_{0}\right.$ or $\left.k_{0}\right)$ : numerical example of a shallow tunnel. a) Back analysis of $\lambda_{0}$; b) Back analysis of $k_{0}$. 

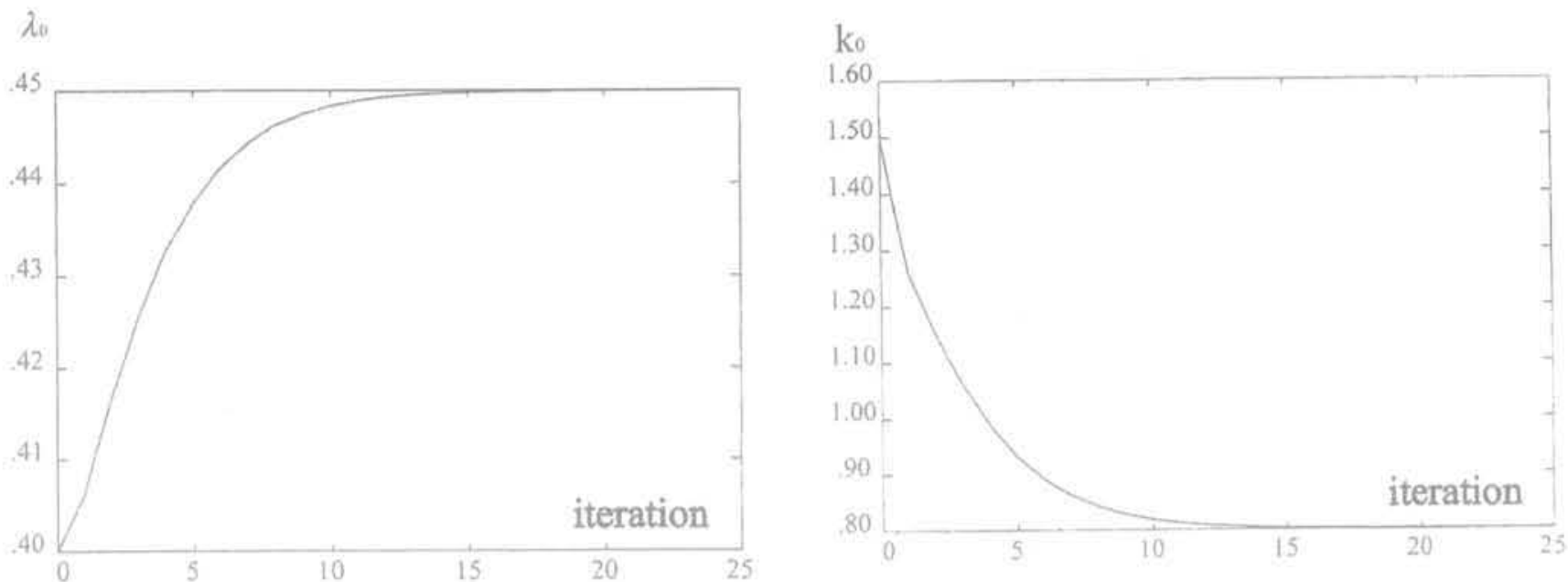

FIG. 13 Convergence de $\lambda_{0}$ et $k_{0}$ : exemple numérique d'un tunnel peu profond. Convergence of $\lambda_{n}$ and $k_{0}$ : numerical example of a shallow tunnel.

ko
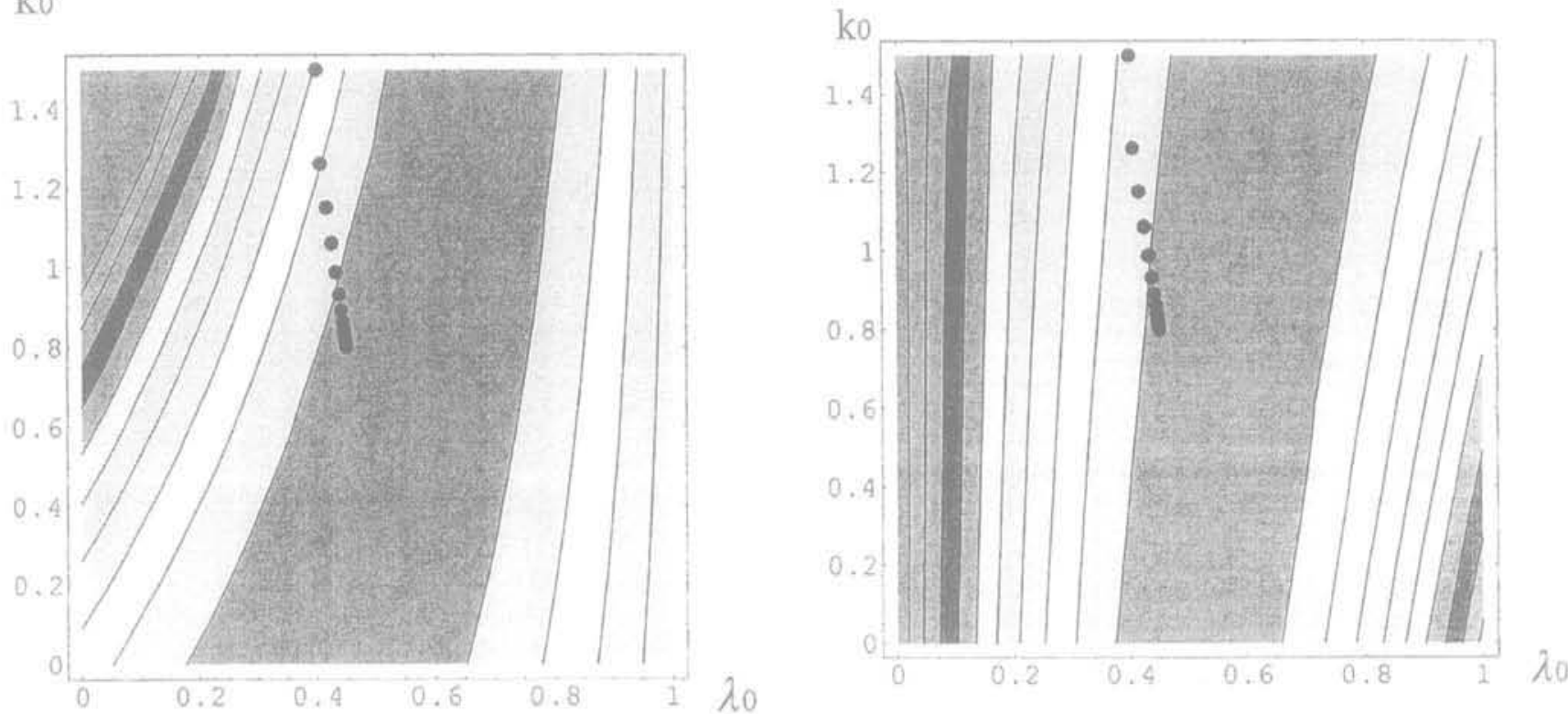

Fig. 14 Chemin de la rétro-analyse de $\lambda_{0}$ et $k_{0}$ (valeurs initiales: $\lambda_{p}=0,4, k_{0}=1,5$ ) : exemple numérique d'un tunnel peu profond. a) Contours des isovaleurs de $F$ à l'instant $t_{0^{*}}$ avec la mesure $u^{m}$; b) Contours des isovaleurs de $F$ à l'instant $t_{r}$ avec la mesure $\Delta \mathbf{u}^{m}$.

Routine of the back analysis of $\lambda_{1}$ and $k_{0}$ (initial values: $\lambda_{0}=0.4, k_{0}=1.5$ ) : numerical example of a shallow tunnel. a) Contours of isovalues of $F$ at instant $t_{0}$, with the measurement $u^{m}$; b) Contours of isovalues of $F$ at instant $t_{p}$, with the measurement $\Delta u^{m}$.

\section{Application à un ourrage réel: rétro-analyse de la discontinuité dans la fondation du barrage Água Vermelha}

Le barrage Água Vermelha est construit en 1979 sur le Rio Grande au Brésil. Le barrage principal est un barrage comportant deux remblais de terre homogène compacte, de part et d'autre d'une partie centrale, en structure de béton. Cette partie centrale se compose du mur droit de transition, du barrage poids, des structures de prise d'eau et de la centrale, du mur central,
Ici la caractéristique géologique qui peut poser problème est la discontinuité avec remplissage d'argile dans la fondation du déversoir. Elle parait au niveau $307,3 \mathrm{~m}$ (voir figure 15) dans le basalte dense. D'après. les tests de cisaillement direct in situ pour la résistance, l'angle de frottement de la discontinuité serait de $28^{\circ}$, et la cohésion de $55 \mathrm{KPa}$ (CESP, 1973). La surveillance de cette fondation a donc été faire avec beaucoup de soin.

Pour notre étude de rétro-analyse, on va analyser une coupe de la section du déversoir, la coupe "VS-8n (Fig. 15), dont les mesures de déplacements par extensomètres et pendules pendant la période de mise en eau sont listées dans le tableau II (EH - extensomètre, PD, Pl-pendule).

Pour analyser le comportement et la sécurité du barrage, il faut bien vérifier les paramètres mécaniques de la discontinuité. On va appliquer la méthode de 


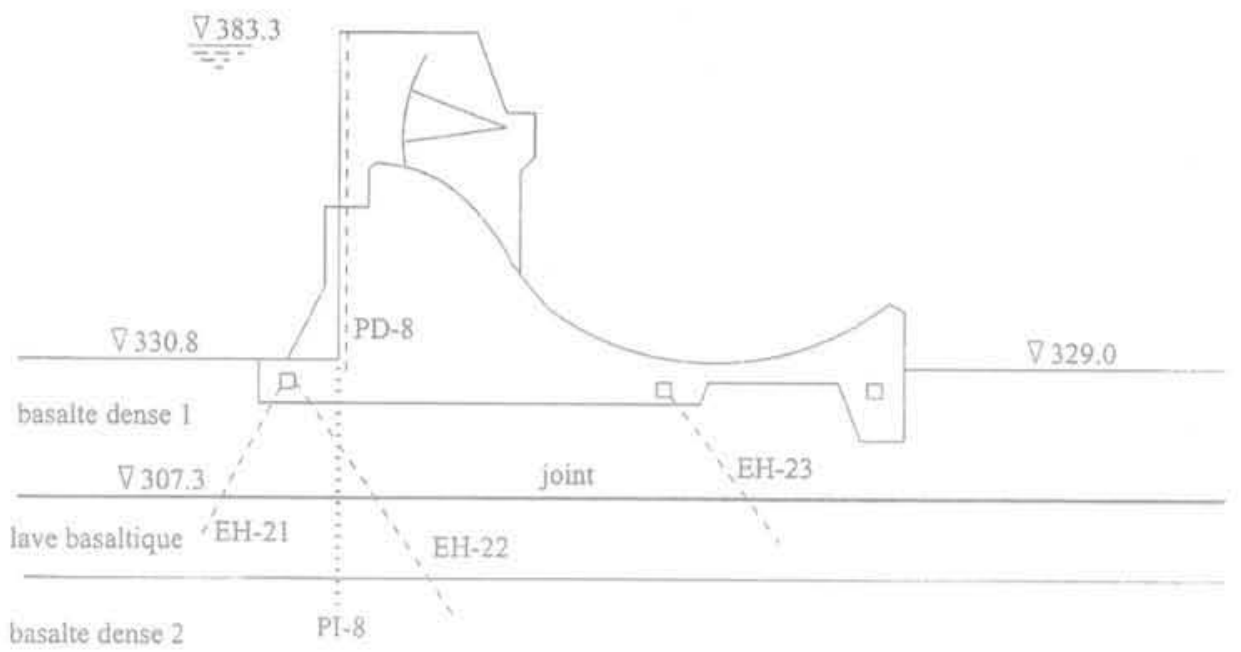

FG. 15 Coupe de la section du déversoir VS-8: barrage Água Vermelha.

Profile of the spillway section VS-8: Agua Vermelha dam.

TABLEAUII Mesures de déplacement pendant mise en eau du réservoir $(\mathrm{nm})$ : barrage Água Vermelha.

\begin{tabular}{l|c|c|c}
\multirow{2}{*}{ Appareil } & \multicolumn{3}{|c}{ Niveau d'eau (m) } \\
\cline { 2 - 4 } EH-21 & $341,48-360$ & $360-370$ & $370-376$ \\
\hline EH-22 & 0,0 & 0,05 & 0,20 \\
\hline EH-23 & 0,0 & 0,0 & $-0,05$ \\
\hline PD-8 & $-0,20$ & $-0,45$ & $-0,25$ \\
\hline Pl-8 & $-1,0$ & $-0,7$ & $-0,6$ \\
\hline
\end{tabular}

rétro-analyse pour l'identification de ces paramètres, avec l'hypothèse de déformation plane.

La discontinuité est traitée comme un joint élastoplastique avec le modèle élastique de Goodman (Goodman, 1976) associé au modèle de Mohr-Coulomb plastique standard.

Les paramètres mécaniques des matériaux sont obtenus d'après des tests en laboratoire et in situ. comme indiqués dans le tableau III (LNEC, 1975).

TABLEAUIII Paramètres mécaniques des matériaux: barrage Água Vermelha.

$(\gamma$ : poids volumique:

\begin{tabular}{l|c|c|c}
\multicolumn{1}{c|}{ Matériau } & $\begin{array}{c}\text { Module d'Young } \\
\text { (MPa) }\end{array}$ & $v$ & $\begin{array}{c}\gamma \\
\left(T / \mathrm{m}^{3}\right)\end{array}$ \\
\hline Béton & 30000,0 & 0,20 & 2,5 \\
\hline Basalte dense 1 & 10000,0 & 0,20 & 2,9 \\
\hline Lave basaltiqué & 7000,0 & 0,20 & 2,9 \\
\hline Basalte dense 2 & 15000,0 & 0,20 & 2,9 \\
\hline Joint & \multicolumn{3}{|c}{$\phi=28^{\circ}, c=55 \mathrm{KPa}$} \\
\hline
\end{tabular}

\section{1}

\section{Rétro-analyse des paramètres élastiques du joint}

En utilisant les mesures de déplacements relatifs pendant le remplissage du barrage du niveau d'eau $341,48 \mathrm{~m}$ au niveau d'eau $360 \mathrm{~m}$, puis du niveau d'eau $360 \mathrm{~m}$ au niveau d'eau $376 \mathrm{~m}$, les rigidités du joint $k_{n}$ et $k_{\text {s }}$ sont identifiées simultanément. La figure 16 montre la convergence de la rétro-analyse des paramètres $k_{f}$ et $k_{\mathrm{s}}$ pour des valeurs initiales : $k_{n}=4,3 \times 10^{8} \mathrm{~N} / \mathrm{m}^{3}$, $k_{\mathrm{s}}=1,4 \times 10^{8} \mathrm{~N} / \mathrm{m}^{3}$. Avec 25 itérations, les deux paramètres élastiques du joint sont obtenus comme: $k_{n}=$ $7,6 \times 10^{3} \mathrm{~N} / \mathrm{m}^{3}, k=1,0 \times 10^{8} \mathrm{~N} / \mathrm{m}^{3}$. Ces valeurs s'accordent bien avec les résultats obtenus par tests de cisaillement direct in situ, les valeurs du $k_{n}$ obtenues des tests in situ étaient dans la plage $(0,96 \sim 9,6) \times 10^{8} \mathrm{~N} / \mathrm{m}^{3}$ (Silveira et al., 1978).

\section{2}

\section{Rétro-analyse des paramètres plastiques du joint}

Dans la rétro-analyse des paramètres élastiques $k_{n}$ et $k_{\alpha}$ on a remarqué que le joint reste entièrement élastique sous ces chargements. Ainsi, pour pouvoir illustrer la méthode de rétro-analyse en plasticité dans ce problème, on a augmenté artificiellement le niveau d'eau de façon à ce que la plasticité se produise dans le joint. Une analyse directe, avec les paramètres mécaniques du tableau III et les paramètres élastiques du joint obtenus par rétro-analyse et le niveau d'eau augmenté jusqu'à $460 \mathrm{~m}$, a été effectuée pour servir de «mesures » à la rétro-analyse en plasticité. Les déplacements de la partie haute (sur une moitié) de la surface en amont et de la surface horizontale au sommet du barrage sont sélectionnés comme mesures. On cherche à identifier l'angle de frottement $\phi$ et la cohésion $c$ du joint.

Les " mesures » obtenues de l'analyse directe au niveau d'eau $445 \mathrm{~m}$ et $460 \mathrm{~m}$ (la plasticité se présente sur $30 \%$ et $45 \%$ respectivement de la partie du joint sous la base du barrage) sont données dans la rétro-analyse. 
$\mathrm{kn}$

$\mathrm{XI}_{8.00}$

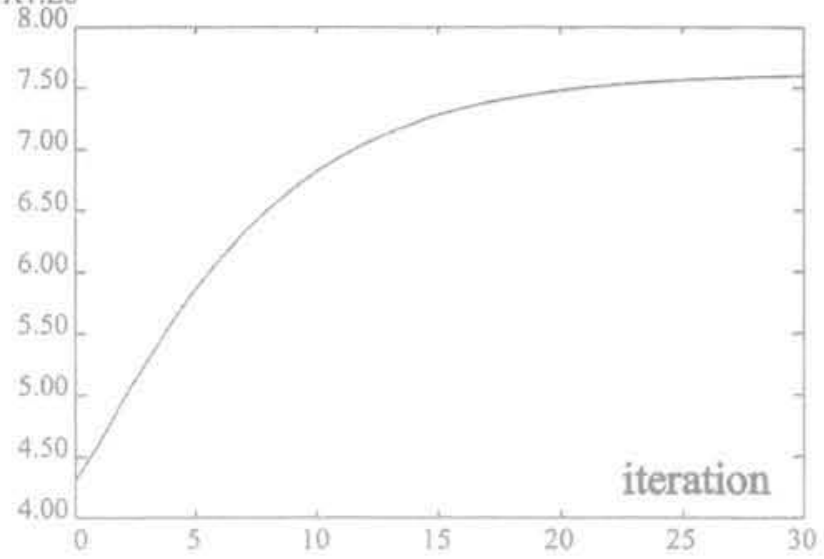

ks

X1.E8

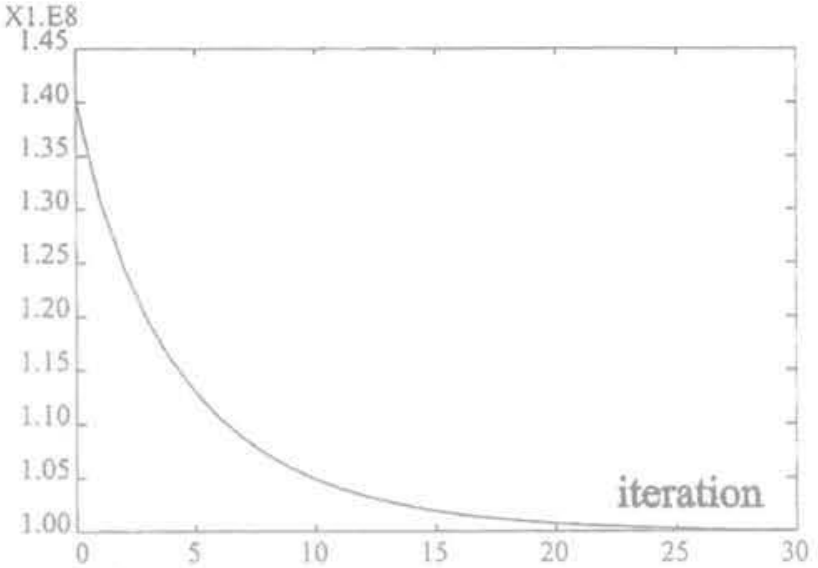

FIG. 16. Convergence d'itération de $k_{\mathrm{n}}$ et $k_{\mathrm{s}}$ : barrage Água Vermelha.

Convergence of $k_{b}$ and $k_{s}$ : the Agua Vermelha dam.

La figure 17 montre la procédure de la rétro-analyse avec les valeurs initiales $\phi=35^{\circ}, c: 35 \mathrm{KPa}$.

Les calculs donnent ainsi une convergence d'itération rapide, même en élasto-plasticité, ce qui illustre l'efficacité de la méthode. Cette application montre aussi la possibilité de rétro-analyser progressivement différents paramètres au cours du chargement: identification des paramètres élastiques par les mesures sous faibles charges, et en utilisant ces paramètres identifiés, identification des paramètres plastiques avec les mesures sous charges supérieures.

\section{5}

\section{Conclusion}

On a proposé une nouvelle mèthode de rétro-analyse basêe sur le thêorème des travaux virtuels (TTV). Pour réaliser la rétro-analyse, un nouveau critère d'identification, la minimisation sur l'erreur en TTV, est établi par comparaison entre le modèle de référence et celui modifié en fonction des mesures obtenues. Puisque le TTV est un principe fondamental de la mécanique, indépendant de la loi de comportemerit, la méthode proposée est puissante, valable pour les problèmes non linéaires en élasto-plasticité. Dans cette rétro-analyse, on a pris le principe de minimisation en utilisant les dérivées de la fonctionnelle coût par rapport aux paramètres inconnus, mais on n'a pas besoin d'obtenir ces dérivées (ce qui est en général très difficile), la méthode est donc simple et efficace. Dans la programmation, on utilise le code du calcul direct comme sous-programme, la programmation de la rétro-analyse est facile (les calculs sont faits avec le logiciel CASTEM2000 du CEA). Avec la nouvelle méthode, on peut identifier des paramètres mécaniques du matériau élastiques ou d'une discontinuité, ainsi que des paramètres de chargement. Pour rétroanalyser plusieurs paramètres il faut plusieurs séries de mesures. En revanche, cette méthode ne semble pas adaptée à l'identification d'une géométrie.

Les cas réels présentent bien entendu une plus grande complexité que les modèles numériques testés ici. Nous avons choisi un cas vécu pour illustrer la faisabilité de la méthode et les résultats obtenus sont assez concluants. Dans la pratique, cette méthode peut $\varphi$

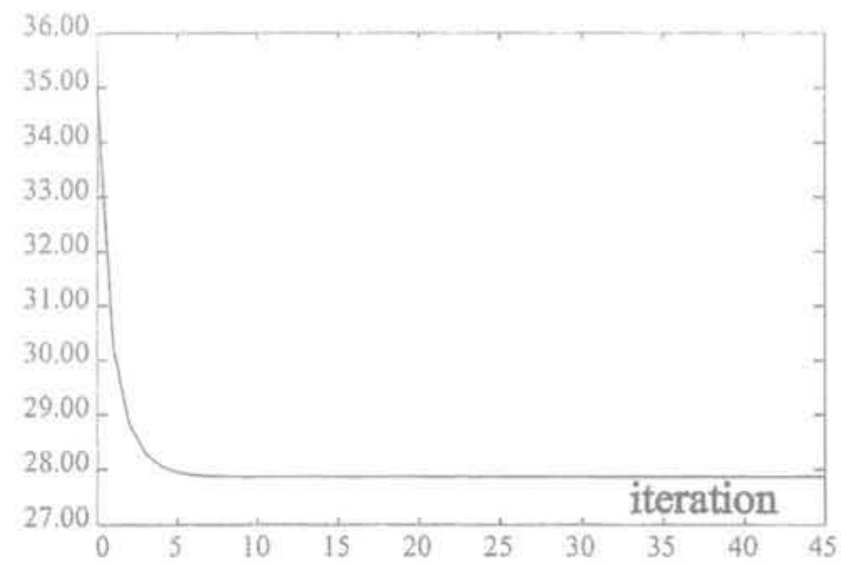

$\mathrm{C}$

X1. 54

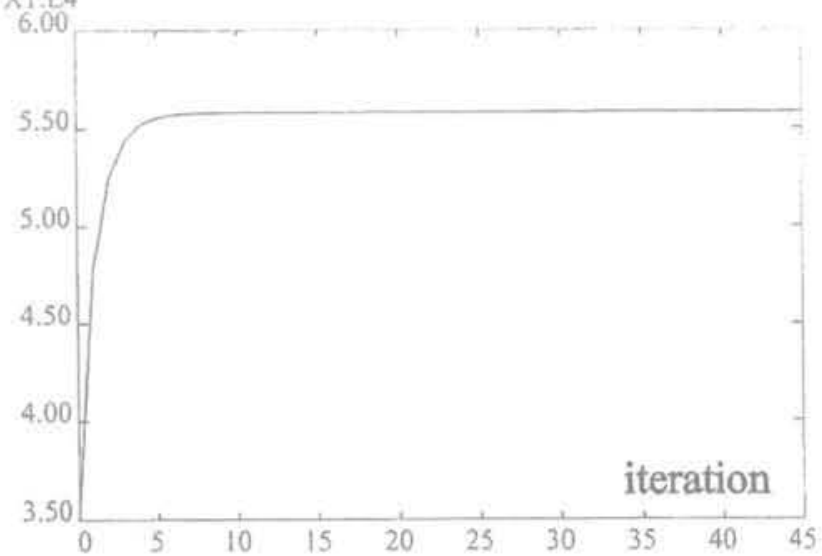

FIG. 17 Rétro-analyse de $\phi$ et c de la discontinuité : barrage Áqua Vermelha. Back analysis of $\phi$ and $c$ of the discontinuity: the Ägua Vermelha dam. 
être utile pour le suivi d'un chantier, dans la mesure où on peut disposer des mesures au cours des chargements, pour pouvoir rétro-analyser successivement les différents paramètres. Aux faibles niveaux de chargement on peut identifier les paramètres élastiques, ensuite, on peut identifier des paramètres plastiques à des niveaux de chargement supérieur.

L'application de la méthode à des problèmes élastovisco-plastiques ou autres phénomènes différés (par exemple avec l'écoulement d'eau interstitielle) est une perspective qui reste encore à explorer. il convient dans ce cas de voir si la méthode peut être utilisée telle quelle, ou si elle ne nécessite pas le cas échéant des modifications. En réalité, les mesures sont toujours affectées d'erreurs, le développement de cette méthode avec une approche statistique, pour obtenir des résultats dans un sens stochastique est une autre voie de recherche.

\section{REMERCIEMENTS}

Les auteurs sont redevables à Andrei Constantinescu pour ses nombreuses et fructucuses discussions au cours de ce travail, ains qu'à Luis Ribeiro e Sousa qui leur a fourni les mesures sur le tunnel de Cidade Universitária et sur le barrage Água Vermelha.

\section{Bibliographie}

AFTES - « Stabilité des turnels par la méthode convergence-confinement $x$. Tunnels et ouvrages souterrains, 32. 1979, p. $70-76$

Akutagawa S, - A back analysis program system for geomechanics applications. PhD thesis, The University of Queensland, 1991.

Arnaudiés J.M. Fraysse H. - Cours de mathématiques - 2: Analysé. Dunod Université, 1989

CESP - Barragem de Áqua Vermelha Deformabilidade e cisalhamento direto in situ em estruturas tipo junta-falha. Companhia Energética de São Paulo. Sào Paulo, 1973.

Cividini A.. Jurina 1. Gioda G. - a Some aspects of "characterization" problems in geomechanics 1 , Int. J. Rock mech. min. sci. \& geomech. abstr., 18, 1981 , p. $487-503$.

Constantinescu A - Sur Pidentification des modules élastiques. PhD thesis, Ecole polytechnique, Plaiseau, France, 1994.

Deng D. Nquven-Minh D. - « Back analysis on mechanical behaviour of joints, using a new method based on virtual work principle n. Desai C.S. Kundu T. Harpalani S. Contractor D., Kemeny J. (eds). Computer methods and advances in geomechanics. Proceedings of the 10th int cong. of IACMAG, vol, 2, Balkema, 2001, p. 915-919.

Deng D.. Nguven-Minh D. Constantinescu A. Vieira A., Sousa L.R. - « Back-analy- sis method for loading parameters in "CV-CF" tunnel calculation - application to a case history w. Travaux en souterrain, ambitions et réalités, AFTES - Journées d'études internationales de Paris, Spécifique JLP, 1999, p. 189-195.

Gioda G. Jurina L. - " Numerical identification of soil-structure interaction pressures v). International journal for numerical and analytical methods in geomechanics, 5, 1981, p. 33-56.

Gloda G., Sakurai S. - « Black analysis procedures for the interpretation of field measurements in geomechanics. p International journal for numerical and analytical methods in geomechanics, 11, 1987. p. 555-583.

Goodman R.E. - Methods of geological engineering in discontinuous rocks. West Publishing Compagny. 1976.

Gudehus G. - Finite Elements in Geomechanics. John Wiley \& Sons. 1977.

Ledesma A., Gens A., Alonso E. E. - « Estimation of parameters in geotechnical back analysis - l. Maximum Likelihood approach, $\mathrm{D}$ Computers and geotechnics 18. 1996, p. 1-27.

LNEC - $\mu$ Estudo da barragen de áqua Vermelha pelo método elementos finitos, i) LNEC report. Lisboa, Portucial, 1975.

Panet M. - Le calcul des tunneis par Ja méthode convergence-confinement. École nationale de ponts et chaussées. Paris, 1995.
Press W. Teukolsky S A Vetterling W.T. Flannery B.P.- Numerical recipes in FORTRAN, the art of scientific computing. Cambridge University Press, 1992.

Sakurai S. - «Back analysis in rock engrg. Hudson J.A. fedi. Comprehensive rock engrg., Pergamon, 1993, p.543-569.

Sakurai S., Akutagawa S. Tokudome O. $\alpha$ Characterization of yield function and plastic potential function by back analysis. $x$ Siriwardane and Zaman (eds). Computeur methods and advances in geomechanics, Balkema. 1994; p.2011. 2016

Sakurai S., Takeuchi K. - "Back analysis of measured displacements of tunnels $n$. Rock mechanics and rock engineering. 16. 1983, p. 173-180.

Shahrour I., Ghorbanbeigi S. - " Analvse tridimensionnelle du comportement des tunnels dans des terrains meubles. Revue francaise de qéotechnique, 76 . 1996. p. 17-22.

Silveira J.F., Miya S., Yendo M. - « Geome chanical parameters computed from instrumentation measurements at Agua Vermelha dam foundation. D Kanii M.A. and Abrahao R.A. (eds), International symposium on rock mechanics related to dam foundation. Rio de Janeiro, Brazil 1978, p. 11.103-11.121

Yang L. - Reverse theory and practice in geotechnical engineering (in Chinese) Science Press, Beijing, 1996. 